\section{Hormonal crisis!}

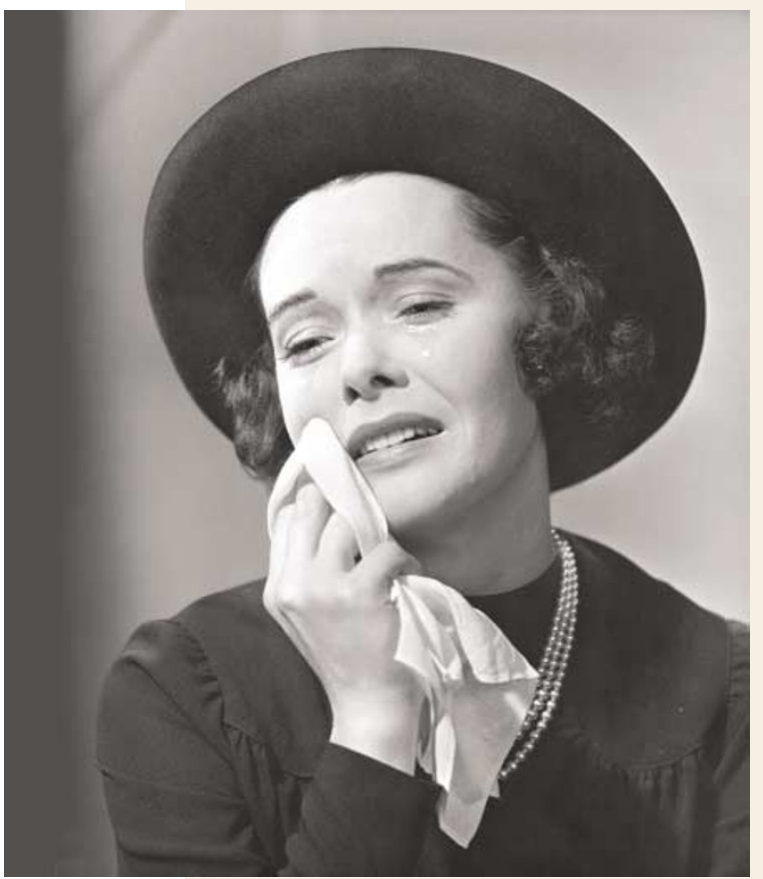

Oestrogen deficiency is known to negatively regulate the immune system, and can lead to the development of autoimmune diseases. As oestrogen levels are naturally highest in females, the effects of deficiency are more pronounced in females compared with males; in addition, these effects might be expected to increase with age, as natural levels of oestrogen decline. Both oestrogen receptors $\alpha$ and $\beta$ (ER $\alpha$ and $E R \beta)$ are known to be involved in regulating the immune system. Now, Shim et al. report in Proceedings of the National Academy of Sciences that ageing does indeed have a pronounced effect on the immune system in $\mathrm{Er} \beta^{-1-}$ mice. This results in myeloproliferative disease, which resembles human late-stage chronic myeloid leukaemia (CML) — known as lymphoid blast crisis.

By 1.5 years of age, $\operatorname{Er} \beta^{-/-}$mice particularly the females - had greatly enlarged spleens. Hyperplasia in bone marrow, which was also observed, was probably the cause of the spleen enlargement. There was also a high rate of leukocyte infiltration - mainly granulocytes and some B lymphocytes into the liver and lung, which showed a myeloproliferative-like syndrome.

There was an increase in the number of neutrophils and monocytes in the blood, and there was evidence of lymphoid blast crisis in some of the $\operatorname{Er} \beta^{-1-}$ mice. Further analysis of the bone-marrow cells by flow cytometry showed that the total number of granulocytes increased by $15-30 \%$.

These results indicate that, as $\mathrm{Er}^{-/-}$mice age, they develop myeloproliferative disease. Normal haematopoiesis is only possible if both copies of $\operatorname{Er} \beta$ are present, as mice that were heterozygous for $\operatorname{Er} \beta$ also developed the myeloproliferative syndrome. So, mutations in the $E R \beta$ gene in humans might be responsible for causing myeloproliferative diseases such as $\mathrm{CML}$, but this requires further investigation. If confirmed, ER $\beta$ agonists might be effective in the treatment of this disease.

Sandra Clark

(0) References and links ORIGINAL RESEARCH PAPER Shim, G.-J. et al.

Disruption of the estrogen receptor $\beta$ gene in mice causes myeloproliferative disease resembling chronic myeloid leukemia with lymphoid blast crisis. Proc. Natl Acad. Sci. USA early edition 9 May 2003 (doi:10.1073/pnas.073 1830100)

\section{DRUG DELIVERY}

\section{Measuring the matrix}

Most tumours have a dense extracellular matrix that inhibits drug diffusion and serves as a barrier to drug delivery. One of the key components of this barrier is a dense collagen meshwork, although the structure and content of the collagen networks varies between tumour types. Brown et al. have used a non-invasive imaging technique to quantify its density in vivo, allowing researchers to estimate the penetrability of tumours to molecular therapeutics.

Brown et al. used a principle called second harmonic generation (SHG) - an intrinsic fluorescent signal that can be detected without the addition of dyes or other reagents to obtain high-resolution three-dimensional images of fibrillar collagen in vivo. SHG imaging allowed the authors to view and measure the density of fibrillar structures in different tumour types in mice (image shows SHG signal of collagen in red and cancer cell nuclei in green).
The SHG signal was found to vary among tumour types and was correlated with collagen content, as confirmed by other methods such as immunohistochemical staining of tissue sections. When the authors applied collagenase enzymes to mouse melanomas and imaged them in vivo, the SHG signal gradually faded, indicating the breakdown of the collagen matrix. Furthermore, as the SHG signal decreased, the diffusion co-efficient of a labelled molecular probe increased.

The hormone relaxin, which, among other functions, induces cells to produce matrix metalloproteinases (MMPs), also induced matrix changes that could be detected by SHG. SHG imaging showed that when mice were treated with relaxin, the average length and brightness of pre-existing fibres decreased significantly. Furthermore, the diffusion coefficients of fluorescent molecular probes increased, indicating a 'loosening' of the extracellular matrix. The authors concluded that the matrix of tumours in relaxin-treated mice had a more porous structure, leading to increased molecular mobility.

This simple and rapid technique could be used to determine the density of a tumour's collagen meshwork and estimate its potential for drug delivery, obviating the need for biopsies, tissue sectioning or staining. SHG has also provided insight into the mechanisms by which relaxin alters the tumour matrix, providing a new technology to evaluate alternative strategies for modifying the tumour extracellular matrix.

Kristine Novak

(Q) References and links

ORIGiNAL RESEARCH PAPER Brown, E. et al. Dynamic imaging of collagen and its modulation in tumours in vivo, using second harmonic generation Nature Med. 9, 796-801 (2003) FURTHER READING Jain, R. K., Munn, L. I. \& Fukumura, D.
Dissecting tumour pathology using intravital microscopy. Nature Dissecting tumour pathology using
Rev. Cancer 2, 266-276 (2002) WEB SITE

Rakesh Jain's lab: http://steele.mgh.harvard.edu

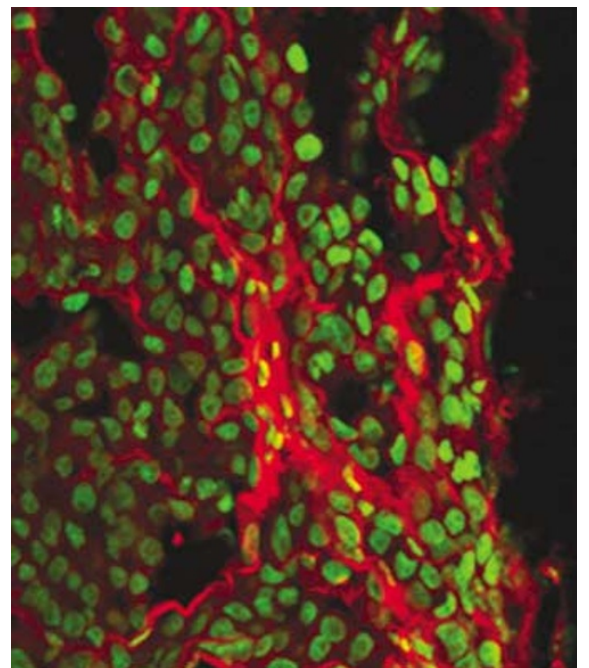

